\title{
A Geometrical Approach of the Levinson Algorithm for Block Toeplitz Matrices
}

\author{
J.G Marcano $^{1}$, M.D. Morán ${ }^{2}$ \\ ${ }^{1}$ Departamento de Matemáticas, Facultad de Ciencias y Tecnología, Universidad de Carabobo, Venezuela \\ ${ }^{2}$ Escuela de Matemática, Facultad de Ciencias, Universidad Central de Venezuela, Venezuela
}

\begin{abstract}
In this paper we obtain a version of the Levinson algorithm for block Toeplitz matrices in an infinite dimensional setting from a geometrical approach. With this methodology we obtain a sequence of operators in the Levinson recurrences whose norms in geometric terms represent angles between subspaces. Additionally, under this geometric framework a block LU decomposition for a block Toeplitz matrix is obtained.
\end{abstract}

Keywords Levinson algorithm, Block Toeplitz matrix, Triangular decomposition, Angles between subspaces.

AMS 2010 subject classifications 15A23, 15B05.

DOI: $10.19139 /$ soic-2310-5070-734

\section{Introduction}

The Levinson algorithm (cf. [20]) is a very important result and has been widely used in prediction and signal processing theory (cf. [5], [13], [21], [22], [26] and [27]). In the infinite dimensional setting, the problem of linear prediction has applications in the context of continuous time processes and for large numbers of time series (cf. [3] and [4]). A first matrix version of the Levinson algorithm was obtained by Whittle (cf. [33]) and later by Wiggins and Robinson (cf. [34]). Matrix versions of this algorithm are also discussed in (cf. [10], [11], [12], [15] and [28]). An extension to the infinite dimensional setting is found in (cf. [16]).

The Levinson recurrences are related to orthogonal polynomials in the unitary circle (cf. [11] and [17]). Also these recurrence are related with the moment problem. From it arises development in theory function, in spectral representation of operator and in statistics. In these recurrences appear an explicit parameters sequence that can be identified with the partial autocorrelation coefficients (cf. [3], [7], [10] and [28]), the reflection coefficients in geophysics (cf. [8] and [16]) and the Schur parameters in analytical functions (cf. [1], [31] and [32]). In the operator theory they are known as choice sequences. These coefficients are very important in the theory of scalar stochastic processes since they allow to characterize the autocorrelation coefficients of the process. An important application of this characterization was the development of a new spectral estimation technique known as the Burg maximum entropy method (cf. [6] and [28]). An extension of this technique to multivariate processes was studied in [29]. In [23] this concept is generalized to the Krein entropy. The partial autocorrelation coefficients are also related to dilation matrices of a stochastic process, so (cf. [14]) proposed the introduction of a new vision of stochastic process through geometry induced by dilation.

The Levinson algorithm is also used in the estimation of the coefficients of the autoregressive linear filter. In this case we need to solve the system of linear equation, $T_{p} X_{p}=Y_{p}$, where $T_{p}$ is a Toeplitz matrix. For

\footnotetext{
*Correspondence to: J.G Marcano (Email:jmarcano@uc.edu.ve). Departamento de Matemáticas, Facultad de Ciencias y Tecnología, Universidad de Carabobo, Venezuela.
}

ISSN 2310-5070 (online) ISSN 2311-004X (print)

Copyright (C) 2019 International Academic Press 
this, it is necessary to obtain the LU decomposition of $T_{p}$. In [9], this decomposition is used to demonstrate that the maximum entropy density subject to the first $p+1$ autocovariance matrices is the spectrum of a multivariate autoregressive process of $p-t h$ order. Problems involving Toeplitz linear equation arise in several applications, e.g. in the prediction of stationary processes, in inverse scattering problem, in buffer analysis for data communication system, among others (cf. [5], [18] and [27]). In [24] and [25] some previous results are obtained using orthogonal decomposition for the finite dimensional case.

The main contribution of this work is to obtain a new version of the Levinson algorithm for block Toeplitz matrices in an infinite dimensional setting using orthogonal decomposition. The parameters obtained in this algorithm are a sequence of contractive operators whose norm can be expressed as angles between subspaces, similar to the finite dimensional case. Also, we obtain a block LU decomposition for block Toeplitz matrices where the triangular matrices can be obtained through Levinson recurrences.

Additionally, our methodology could be useful to solve extension problems in statistics and for prediction problems in an infinite dimensional context (cf. [10], [11], [12], [15], [16], [19], [20], [28], [33] and [34]).

The scheme to obtain the Levinson algorithm is as follows: the data is a sequence of bounded operators $\left\{R_{k}\right\}_{k=0}^{p}$, defined in a separable Hilbert space $\mathcal{G}$. More specifically, based on the sequence of bounded operators $\left\{R_{k}\right\}_{k=0}^{p}$, we built a Hilbert space $\mathcal{H}_{p}$ and a surjective isometry $V_{p}: \mathcal{D}_{p} \rightarrow \mathcal{R}_{p}$, where $\mathcal{D}_{p}, \mathcal{R}_{p}$ are two closed subspaces of $\mathcal{H}_{p}$. In this sense, the defect spaces of the isometry $V_{p}$ are $\mathcal{N}_{p}=\mathcal{H}_{p} \ominus \mathcal{D}_{p}, \mathcal{M}_{p}=\mathcal{H}_{p} \ominus \mathcal{R}_{p}$. Then, the Levinson recurrences are obtained as a consequence of the orthogonal decompositions where the subspaces in this decompositions are the defect spaces.

The paper is organized as follows: in the second section; we introduce some notations and preliminary results; in the third section, we obtain a block LU decomposition for a block Toeplitz matrix; in fourth section, we state the main result of this work and in the last section, we present the conclusions and discussions.

\section{Preliminaries}

First we are going to introduce the notation that we will be using in this work. Denote by $\mathbb{N}$ and $\mathbb{Z}$ to the sets of the natural numbers and the integers respectively. We will use the symbols $\mathbb{R}$ and $\mathbb{C}$ to denote the set of real and complex numbers respectively, $\mathbb{D}$ will denote the open unit disk in the complex plane, that is, $\mathbb{D}:=\{z \in \mathbb{C}:|z|<1\}$. The unitary circle, the boundary of $\mathbb{D}$, will be denoted by $\mathbb{T}$. Define $e_{k}$ by $e_{k}(\zeta):=\zeta^{k}, \zeta \in \mathbb{T}, k \in \mathbb{Z}$. Denote, as usual, the set of all bounded linear operator acting in the Hilbert space $\mathcal{H}$ as $\mathcal{L}(\mathcal{H})$. By 1 we indicate either the scalar unit or the identity operator depending on context.

Next we explicitly explain how to build the Hilbert space $\mathcal{H}_{p}$, the subspaces $\mathcal{D}_{p}$ and $\mathcal{R}_{p}$ and a surjective isometry $V_{p}: \mathcal{D}_{p} \rightarrow \mathcal{R}_{p}$. The defect spaces $\mathcal{N}_{p}$ and $\mathcal{M}_{p}$ are also calculated explicitly. For this, we need that the sequence of bounded linear operators $\left\{R_{k}\right\}_{k=0}^{p}$ verifies certain conditions.

Given a sequence of bounded linear operator $1, R_{1}, \cdots, R_{p}$ acting in the separable Hilbert space $\mathcal{G}$, define $R_{-k}=R_{k}^{*}$, for $k=1, \cdots, p$ and we say that the sequence is strictly positive definite if, and only if,

$$
\sum_{n=0}^{p} \sum_{m=0}^{p}\left\langle R_{m-n} h_{n}, h_{m}\right\rangle_{\mathcal{G}}>0
$$

for all not null sequence $\left\{h_{k}\right\}_{k=0}^{p} \subset \mathcal{G}$.

Now, we stand for $T_{k}, k=0,1, \cdots, p$ the bounded operator $T_{k}: \mathcal{G}^{k+1} \rightarrow \mathcal{G}^{k+1}$ defined by

$$
T_{k}=\left(\begin{array}{cccc}
1 & R_{-1} & \cdots & R_{-k} \\
\vdots & \vdots & \cdots & \vdots \\
R_{k} & R_{k-1} & \cdots & 1
\end{array}\right)
$$

Therefore (1) is equivalent to that the bounded operator $T_{p}$ is strictly positive.

Note that, if the bounded operator $T_{p}$ is strictly positive, then the bounded operators $T_{k}, k=0,1, \cdots, p-1$ are also strictly positive. In the following $\gamma_{k}=\left\{\gamma_{i j}^{k}\right\}_{i, j=0,1, \cdots, k}$ stand for the inverse of $T_{k}$. We know that the 
bounded operators $\gamma_{k}, k=0,1, \cdots, p$ are strictly positive because the bounded operators $T_{k}, k=0,1, \cdots, p$ are strictly positive. Hence, the bounded operators $\gamma_{00}^{k}$ and $\gamma_{k k}^{k}$ for $k=0,1, \cdots, p$ are strictly positive since they are the compression of the operators $\gamma_{k}$ to suitable subspaces of $\mathcal{G}^{k+1}$.

Let's suppose from now on, that the sequence of bounded operators $\left\{R_{k}\right\}_{k=-p}^{p}$ satisfies (1).

Define $\mathcal{E}_{p}=\left\{\phi=\sum_{k=0}^{p} e_{k} \xi_{k}: \xi_{k} \in \mathcal{G}, k=1, \cdots, p\right\}$ as the set of all analytical trigonometrical polynomials of degree less or equal $p$, in $\mathbb{T}$ with values in a Hilbert space $\mathcal{G}$.

Define the inner product in $\mathcal{E}_{p}$ by:

$$
\begin{aligned}
\left\langle\sum_{n=0}^{p} e_{n} f_{n}, \sum_{m=0}^{p} e_{m} g_{m}\right\rangle_{p} & =\sum_{m=0}^{p} \sum_{n=0}^{p}\left\langle R_{m-n} f_{n}, g_{m}\right\rangle_{\mathcal{G}} \\
& =\left\langle T_{p}\left(\begin{array}{c}
f_{0} \\
\vdots \\
f_{p}
\end{array}\right),\left(\begin{array}{c}
g_{0} \\
\vdots \\
g_{p}
\end{array}\right)\right\rangle_{\mathcal{G}^{p+1}} .
\end{aligned}
$$

The space $\left(\mathcal{E}_{p},\langle,\rangle_{p}\right)$ is a Hilbert space. Indeed, we obtain this result from the fact that the operator $I_{p}$ : $\left(\mathcal{E}_{p},\langle., .\rangle_{p}\right) \rightarrow \mathcal{G}^{p+1}$ defined by $I_{p}\left(\sum e_{k} h_{k}\right)=\left(h_{0}, h_{1} \cdots, h_{p}\right)$ is a bounded and invertible.

Set $\quad \mathcal{D}_{p}=\left\{\sum_{k=0}^{p-1} e_{k} \xi_{k}: \xi_{j} \in \mathcal{G}, j=0,1, \ldots, p-1\right\}, \quad \mathcal{R}_{p}=\left\{\sum_{k=1}^{p} e_{k} \xi_{j}: \xi_{j} \in \mathcal{G}, k=1, \ldots, p\right\} \quad$ and $V_{p}: \mathcal{D}_{p} \rightarrow \mathcal{R}_{p}$ the map defined as the linear extension of $V_{p}\left(e_{k} \xi\right)=e_{k+1} \xi$.

Let $L_{\mathcal{G}}^{2}$ as usual,

$$
L_{\mathcal{G}}^{2}=\left\{f: \mathbb{T} \rightarrow \mathcal{G} \mid f \text { measurable and } \frac{1}{2 \pi} \int_{0}^{2 \pi}\left\|f\left(e^{i t}\right)\right\|_{\mathcal{G}}^{2} d t<\infty\right\} .
$$

Clearly, $L_{\mathcal{G}}^{2}$ is a Hilbert space with the standard inner product

$$
\langle f, g\rangle_{L_{\mathcal{G}}^{2}}=\frac{1}{2 \pi} \int_{0}^{2 \pi}\left\langle f\left(e^{i t}\right), g\left(e^{i t}\right)\right\rangle_{\mathcal{G}} d t .
$$

For $k \in \mathbb{Z}$, let $\mathcal{G}_{k}$ the subspace of $L_{\mathcal{G}}^{2}$ of the functions of the form $e_{k} a(a \in \mathcal{G})$. It can be seen in [30] such that $\mathcal{G}_{i}$ is orthogonal to $\mathcal{G}_{j}$, if $i \neq j$. Moreover,

$$
L_{\mathcal{G}}^{2}=\bigoplus_{-\infty}^{\infty} \mathcal{G}_{k}
$$

and

$$
\|a\|_{\mathcal{G}}=\left\|e_{k} a\right\|_{L_{\mathcal{G}}^{2}} \text {. }
$$

Let $\Gamma_{p}:\left(\mathcal{E}_{p}(\mathcal{G}),\langle., .\rangle_{p}\right) \rightarrow\left(\mathcal{E}_{p},\langle., .\rangle_{L_{\mathcal{G}}^{2}}\right)$ be the map defined by

$$
\Gamma_{p}(f)=\sum_{k=0}^{p} e_{k} \sum_{s=0}^{p} R_{k-s} f_{s}, f \in \mathcal{E}_{p} .
$$

Clearly $\Gamma_{p}$, is well defined.

The next result shows that $V_{p}$ is a surjective isometry and that the operator $\Gamma_{p}$ is bicontinuos.

Proposition 1 (a) $V_{p}$ is a surjective isometry acting in $\left(\mathcal{E}_{p},\langle,\rangle_{p}\right)$.

(b) The operator $\Gamma_{p}$ satisfies the equality $\left\langle\Gamma_{p} f, g\right\rangle_{L_{\mathcal{G}}^{2}}=\langle f, g\rangle_{p}$.

(c) The operator $\Gamma_{p}$ is bicontinuos.

(d) The defect spaces of $V_{p}, \mathcal{N}_{p}=\mathcal{E}_{p} \ominus \mathcal{D}_{p}$ y $\mathcal{M}_{p}=\mathcal{E}_{p} \ominus \mathcal{R}_{p}$ are generated by elements of the form $\Gamma_{p}^{-1}\left(e_{p} x\right)$ and $\Gamma_{p}^{-1}\left(e_{0} x\right), x \in \mathcal{G}$ respectively. Moreover,

$$
\begin{gathered}
\Gamma_{p}^{-1}\left(e_{p} x\right)=\left(e_{0} \gamma_{0 p}^{p}+\cdots+e_{p} \gamma_{p p}^{p}\right) x \\
\Gamma_{p}^{-1}\left(e_{0} x\right)=\left(e_{0} \gamma_{00}^{p}+\cdots+e_{p} \gamma_{p 0}^{p}\right) x
\end{gathered}
$$


Proof: The statement (a) is very easy since $R_{(m+1)-(n+1)}=R_{m-n}$.

In order to see the statement (b), let $f=\sum_{k=0}^{p} e_{k} f_{k}, g=\sum_{k=0}^{p} e_{k} g_{k} \in \mathcal{E}_{p}$, thus

$$
\begin{aligned}
\left\langle\Gamma_{p} f, g\right\rangle_{L_{\mathcal{G}}^{2}} & =\frac{1}{2 \pi} \int_{0}^{2 \pi}\left\langle\sum_{l=0}^{p} e_{l} \sum_{m=0}^{p} R_{l-m} f_{m}, \sum_{k=0}^{p} e_{k} g_{k}\right\rangle_{\mathcal{G}} d t \\
& =\langle f, g\rangle_{p}
\end{aligned}
$$

To prove (c), we note that the operator $J_{p}:\left(\mathcal{E}_{p}(\mathcal{G}),\langle., .\rangle_{L_{\mathcal{G}}^{2}}\right) \rightarrow \mathcal{G}^{p+1}$ defined by $J_{p}\left(\sum_{k=0}^{p} e_{k} h_{k}\right)=$ $\left(h_{0}, h_{1} \cdots, h_{p}\right)$ is a unitary isomorphism. Now,

$$
J_{p}^{*} T_{p} I_{p}\left(\sum_{k=0}^{p} e_{k} h_{k}\right)=\Gamma_{p}\left(\sum_{k=0}^{p} e_{k} h_{k}\right) .
$$

Therefore $\Gamma_{p}=J_{p}^{*} T_{p} I_{p}$ and hence $\Gamma_{p}$ is bicontinuos.

To see the statement (d), let $f \in \mathcal{E}_{p} \ominus \mathcal{D}_{p}$ and $\left\{\alpha_{k}\right\}_{k=1}^{\infty}$ be a complete orthonormal system of the Hilbert space $\mathcal{G}$. From (b) we obtain the classical normal equations, that is, for $j \in \mathbb{N}$,

$$
\left\langle\Gamma_{p} f, e_{k} \alpha_{j}\right\rangle_{L_{\mathcal{G}}^{2}}=\left\langle f, e_{k} \alpha_{j}\right\rangle_{p}=0 \text { for } k=0,1, \ldots, p-1 .
$$

Thus, from (2), for every $j=1,2, \cdots$, there exists $f^{j} \in \mathcal{E}_{p} \ominus \mathcal{D}_{p}$ such that $\Gamma_{p}\left(f^{j}\right)=e_{p} \alpha_{j}$. Therefore, we get the generators of the defect space $\mathcal{N}_{p}: f^{j}=\Gamma_{p}^{-1}\left(e_{p} \alpha_{j}\right), j=1,2, \ldots$

In a similar way, we prove that for every $j \in \mathbb{N}$ there exists $g^{j} \in \mathcal{E}_{p} \ominus \mathcal{R}_{p}$ such that $g^{j}=\Gamma_{p}^{-1}\left(e_{0} \alpha_{j}\right)$.

Finally, (4) can be obtained from $\Gamma_{p}=J_{p}^{*} T_{p} I_{p}$.

\section{Lemma 1}

Let $\Gamma_{p}$ the bicontinuos operator defined by (3) and for $1 \leq j<k \leq p$ let $I_{j, k}:\left(\mathcal{E}_{j},\langle,\rangle_{j}\right) \rightarrow\left(\mathcal{E}_{k},\langle,\rangle_{k}\right)$ the identity operator. The following statements are true:

(a)

$$
\left.P_{\mathcal{E}_{p-1}}^{\mathcal{E}_{p}} \Gamma_{p}\right|_{\mathcal{E}_{p-1}}=\Gamma_{p-1}
$$

where $\left.\Gamma_{p}\right|_{A}$ is the restriction of the operator $\Gamma_{p}$ to the set $A$ and $P_{\mathcal{E}_{p-1}}^{\mathcal{E}_{p}}$ is the orthogonal projection of $\mathcal{E}_{p}$ onto $\mathcal{E}_{p-1}$.

(b) Let $x, y \in \mathcal{E}_{p-1}$. Then,

$$
\langle x, y\rangle_{p}=\left\langle\Gamma_{p} x, y\right\rangle_{L_{\mathcal{G}}^{2}}=\left\langle\Gamma_{p-1} x, y\right\rangle_{L_{\mathcal{G}}^{2}}=\langle x, y\rangle_{p-1} .
$$

(c) For $1 \leq j<k \leq p, I_{j, k}$ is an isometry.

(d) There exists an isometry $J$ such that

$$
\begin{aligned}
& -\mathcal{D}_{p}=\mathcal{E}_{p-1}=J\left(\mathcal{D}_{p-1} \oplus \mathcal{N}_{p-1}\right)=J\left(\mathcal{R}_{p-1} \oplus \mathcal{M}_{p-1}\right)=J\left(V_{p-1} \mathcal{D}_{p-1} \oplus \mathcal{M}_{p-1}\right) \\
& \text { - } \mathcal{R}_{p}=V_{p} \mathcal{D}_{p}=J\left(\mathcal{R}_{p-1} \oplus V_{p-1} \mathcal{N}_{p-1}\right) . \\
& \text { - } \mathcal{E}_{p}=\mathcal{N}_{p} \oplus \mathcal{N}_{p-1} \oplus \cdots \oplus \mathcal{N}_{1} \oplus \mathcal{N}_{0} \\
& \text { - } \mathcal{E}_{p}=V_{p}^{p} \mathcal{M}_{0} \oplus V_{p}^{p-1} \mathcal{M}_{p-1} \oplus \cdots \oplus V_{p} \mathcal{M}_{p-1} \oplus \mathcal{M}_{p}
\end{aligned}
$$

Proof: To see (a), note that $\left(\mathcal{E}_{p-1},\langle,\rangle_{L_{\mathcal{G}}^{2}}\right)$ is a subspace of $\left(\mathcal{E}_{p},\langle,\rangle_{L_{\mathcal{G}}^{2}}\right)$. Hence, from (3) we obtain the result.

The statements (b) and (c) are easy to prove.

The statement (d) is a direct consequence of the above proposition with $J:=I_{p-1, p}$ and from the preceding results. 


\section{A lower and upper triangular factorization of the matrix $T_{p}^{-1}$}

The main result of this section is a lower and upper factorization of the matrix $T_{p}^{-1}$. To get such factorizations we need to obtain complete orthonormal systems of the defect space $\mathcal{M}_{p}$ and $\mathcal{N}_{p}$.

For this, we consider the operator $L_{k}:\left(\mathcal{G},\langle,\rangle_{\mathcal{G}}\right) \rightarrow\left(\mathcal{G}_{k},\langle,\rangle_{L_{\mathcal{G}}^{2}}\right)$ for $k=0, p$ defined by $L_{k} x=e_{k} x, x \in \mathcal{G}$ where $\mathcal{G}_{k}$ are the subspace of $L_{\mathcal{G}}^{2}$ previously introduced. Note that this operators are isometric isomorphisms. Now, let $\widetilde{M}_{p}\left(e^{i t}\right):\left(\mathcal{G},\langle,\rangle_{\mathcal{G}}\right) \rightarrow\left(\mathcal{M}_{p},\langle,\rangle_{p}\right)$ and $\widetilde{N}_{p}\left(e^{i t}\right):\left(\mathcal{G},\langle,\rangle_{\mathcal{G}}\right) \rightarrow\left(\mathcal{N}_{p},\langle,\rangle_{p}\right)$ the operator trigonometric polynomials $\widetilde{M}_{p}\left(e^{i t}\right)=\Gamma_{p}^{-1} L_{0}$ and $\widetilde{N}_{p}\left(e^{i t}\right)=\Gamma_{p}^{-1} L_{p}$ respectively. Clearly this operators are invertible with inverse given by $\widetilde{M}_{p}^{-1}\left(e^{i t}\right)=\left.L_{0}^{*} \Gamma_{p}\right|_{\mathcal{M}_{p}}$ and $\widetilde{N}_{p}^{-1}\left(e^{i t}\right)=\left.L_{p}^{*} \Gamma_{p}\right|_{\mathcal{N}_{p}}$ Thus,

$$
\begin{aligned}
\widetilde{M}_{p}\left(e^{i t}\right) x & =\Gamma_{p}^{-1}\left(e_{0} x\right)=\left(e_{0} \gamma_{00}^{p}+\cdots+e_{p} \gamma_{p 0}^{p}\right) x, \\
\widetilde{N}_{p}\left(e^{i t}\right) x & =\Gamma_{p}^{-1}\left(e_{p} x\right)=\left(e_{0} \gamma_{0 p}^{p}+\cdots+e_{p} \gamma_{p p}^{p}\right) x .
\end{aligned}
$$

From these operators we define the normalized operators trigonometric polynomials $M_{p}\left(e^{i t}\right)$ and $N_{p}\left(e^{i t}\right)$ given by

$$
M_{p}\left(e^{i t}\right) x=\widetilde{M}_{p}\left(e^{i t}\right)\left(\gamma_{00}^{p}\right)^{-1 / 2} x, \quad N_{p}\left(e^{i t}\right) x=\widetilde{N}_{p}\left(e^{i t}\right)\left(\gamma_{p p}^{p}\right)^{-1 / 2} x .
$$

From now on, we write $M_{p}$ and $N_{p}$ instead of $M_{p}\left(e^{i t}\right)$ and $N_{p}\left(e^{i t}\right)$ respectively.

This operators can be obtained from the classical equation (cf. [2], [16], [28] and [35]) using (3), the classical normal equation ((5) for $N_{p}$ ) and from the formula $\Gamma_{p}^{-1}=I_{p}^{*} T_{p}^{-1} J_{p}$.

The following result shows that from these operators can be obtained complete orthonormal systems of the defect space of the isometry $V_{p}$.

Lemma 2

Let $\left\{\alpha_{k}\right\}_{k=1}^{\infty}$ a complete orthonormal system of the separable Hilbert space $\mathcal{G}$. Then,

$$
m_{p}^{k}=M_{p} \alpha_{k} \quad \text { and } \quad n_{p}^{k}=N_{p} \alpha_{k} ; \quad k=1,2, \ldots
$$

are complete orthonormal systems of the defect spaces $\mathcal{M}_{p}$ and $\mathcal{N}_{p}$ respectively.

Proof: The result is a direct consequence of the preceding proposition and (4).

Using a similar proof given in [24] we can to show that the zeros of $n_{p}^{k}$ and $m_{p}^{k}$ for $k=1,2, \ldots$, lie in the open unit disk D and in the exterior of the closed unit disk, respectively.

\section{Lemma 3}

Let $M_{p}$ and $N_{p}$ the operator trigonometric polynomials defined in (6). Then, the following properties are true:

(a) $M_{p}$ and $N_{p}$ are isometric isomorphism.

(b) For all $j, k=1, \cdots, p$ and every $x, y \in \mathcal{G} ;\left\langle N_{j} x, N_{k} y\right\rangle_{p}=\delta_{j k}$ and $\left\langle e_{p-k} M_{k} x, e_{p-j} M_{j} y\right\rangle_{p}=\delta_{j k}$.

Proof: The statement (a) is a direct consequence of the previous lemma.

The statement (b) follows from the orthogonal decompositions $\mathcal{E}_{p}=\mathcal{N}_{0} \oplus \mathcal{N}_{1} \oplus \cdots \oplus \mathcal{N}_{p}$ and $\mathcal{E}_{p}=V_{p}^{p} \mathcal{M}_{0} \oplus$ $V_{p}^{p-1} \mathcal{M}_{p-1} \oplus \cdots \oplus V_{p} \mathcal{M}_{p-1} \oplus \mathcal{M}_{p}$.

Denote $M_{p}$ and $N_{p}$ by

$$
M_{p}=e_{0} M_{p, 0}+\cdots+e_{p} M_{p, p} \text { and } N_{p}=e_{0} N_{p, 0}+\cdots+e_{p} N_{p, p}
$$

and define the matrices $\alpha_{p}$ and $\beta_{p}$ by

$$
\alpha_{p}=\left(\begin{array}{cccc}
M_{p, 0} & \cdots & M_{1,0} & M_{0,0} \\
M_{p, 1} & \cdots & M_{1,1} & 0 \\
\vdots & \vdots & \vdots & \vdots \\
M_{p, p} & \cdots & 0 & 0
\end{array}\right) \text { and } \beta_{p}=\left(\begin{array}{cccc}
N_{0,0} & N_{1,0} & \cdots & N_{p, 0} \\
0 & N_{1,1} & \cdots & N_{p, 1} \\
\vdots & \vdots & \cdots & \vdots \\
0 & 0 & \cdots & N_{p, p}
\end{array}\right)
$$


Since $N_{j, j}, j=0,1, \cdots, p$ are strictly positive,

$$
\beta_{1}=\left(\begin{array}{cc}
1 & N_{1,0} \\
0 & N_{1,1}
\end{array}\right)
$$

is invertible and for $i=2, \cdots, p$

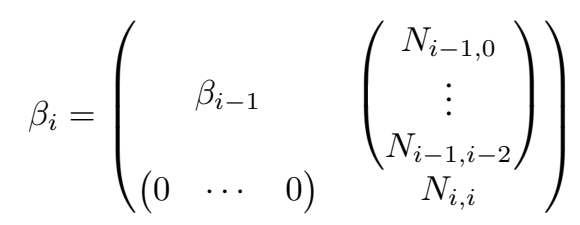

we can prove recursively that $\beta_{i}$ for $i=2, \cdots, p$ are invertible. Analogously, a similar result can be obtained for $\alpha_{i}, i=1, \cdots, p$.

The following result shows a lower and upper triangular factorization of $T_{p}^{-1}$.

\section{Proposition 2}

Let $\alpha_{p}$ and $\beta_{p}$ the matrices defined above. Then,

$$
\beta_{p} \beta_{p}^{*}=T_{p}^{-1}=\alpha_{p} \alpha_{p}^{*}
$$

Proof: Let $f=\left(f_{0}, \cdots, f_{p}\right)^{t}$ and $g=\left(g_{0}, \cdots, g_{p}\right)^{t}$. From the statement (b) of the previous lemma, we have

$$
\begin{aligned}
& \langle f, g\rangle_{\mathcal{G}^{p+1}}=\left\langle f_{0}, g_{0}\right\rangle_{\mathcal{G}}+\cdots+\left\langle f_{p}, g_{p}\right\rangle_{\mathcal{G}} \\
& =\left\langle N_{0} f_{0}, N_{0} g_{0}\right\rangle_{p}+\cdots+\left\langle N_{p} f_{p}, N_{p} g_{p}\right\rangle_{p} \\
& =\left\langle R_{0} N_{0,0} f_{0}, N_{0,0} g_{0}\right\rangle_{\mathcal{G}}+\cdots+\sum_{n=0}^{p} \sum_{m=0}^{p}\left\langle R_{m-n} N_{p, n} f_{p}, N_{p, m} g_{p}\right\rangle_{\mathcal{G}} \\
& =\left\langle T_{p}\left(\begin{array}{c}
N_{0,0} f_{0} \\
0 \\
\vdots \\
0
\end{array}\right),\left(\begin{array}{c}
N_{0,0} g_{0} \\
0 \\
\vdots \\
0
\end{array}\right)\right\rangle_{\mathcal{G}^{p+1}}+\cdots+\left\langle T_{p}\left(\begin{array}{c}
N_{p, 0} f_{p} \\
N_{p, 1} f_{p} \\
\vdots \\
N_{p, p} f_{p}
\end{array}\right),\left(\begin{array}{c}
N_{p, 0} g_{p} \\
N_{p, 1} g_{p} \\
\vdots \\
N_{p, p} g_{p}
\end{array}\right)\right\rangle_{\mathcal{G}^{p+1}} \\
& =\left\langle T_{p}\left(\begin{array}{cccc}
N_{0,0} & N_{1,0} & \cdots & N_{p, 0} \\
0 & N_{1,1} & \cdots & N_{p, 1} \\
\vdots & \vdots & \cdots & \vdots \\
0 & 0 & \cdots & \vdots N_{p, p}
\end{array}\right)\left(\begin{array}{c}
f_{0} \\
f_{1} \\
\vdots \\
f_{p}
\end{array}\right),\left(\begin{array}{cccc}
N_{0,0} & N_{1,0} & \cdots & N_{p, 0} \\
0 & N_{1,1} & \cdots & N_{p, 1} \\
\vdots & \vdots & \cdots & \vdots \\
0 & 0 & \cdots & \vdots \\
0 & N_{p, p}
\end{array}\right)\left(\begin{array}{c}
g_{0} \\
g_{1} \\
\vdots \\
g_{p}
\end{array}\right)\right\rangle_{\mathcal{G}^{p+1}}
\end{aligned}
$$

Thus, $\beta_{p}^{*} T_{p} \beta_{p}=1$, or equivalently, $\beta_{p}^{-1} T_{p}^{-1}\left(\beta_{p}^{*}\right)^{-1}=1$. Hence $\beta_{p} \beta_{p}^{*}=T_{p}^{-1}$ and $\beta_{p}^{*}$ is a lower triangular factorization of $T_{p}^{-1}$.

The upper triangular factorization of $T_{p}^{-1}$ can be obtained from the fact that

$$
\begin{aligned}
& \langle f, g\rangle_{\mathcal{G}^{p+1}}=\left\langle f_{0}, g_{0}\right\rangle_{\mathcal{G}}+\cdots+\left\langle f_{p}, g_{p}\right\rangle_{\mathcal{G}} \\
& \quad=\left\langle M_{p} f_{0}, M_{p} g_{0}\right\rangle_{p}+\cdots+\left\langle M_{0} f_{p}, M_{0} g_{p}\right\rangle_{p}
\end{aligned}
$$

From now on to simplify the notation we write $\gamma_{i j}$ instead of $\gamma_{i j}^{k}$ for $i, j=0,1, \cdots, k ; i, j \neq 0$.

We can see in the next section that the Levinson recurrences is an efficient algorithm to obtain this factorization.

\section{Proposition 3}

Let $\gamma_{k}$ the inverse matrix of $T_{k}$ for $k=0,1, \cdots, p$. Then,

$$
\gamma_{k k}^{-1}=1-A_{k}^{*} \alpha_{k-1} \alpha_{k-1}^{*} A_{k}=1-A_{k}^{*} \beta_{k-1} \beta_{k-1}^{*} A_{k}
$$


and

$$
\left(\gamma_{00}^{k}\right)^{-1}=1-C_{k} \alpha_{k-1} \alpha_{k-1}^{*} C_{k}^{*}=1-C_{k} \beta_{k-1} \beta_{k-1}^{*} C_{k}^{*}
$$

where $A_{k}^{*}=\left[R_{k}, R_{k-1}, \cdots, R_{1}\right]$ and $C_{k}=\left[R_{-1}, R_{-2}, \cdots, R_{-k}\right]$.

Proof: First, note that

$$
T_{k}=\left(\begin{array}{cc}
T_{k-1} & A_{k} \\
A_{k}^{*} & 1
\end{array}\right)=\left(\begin{array}{cc}
1 & C_{k} \\
C_{k}^{*} & T_{k-1}
\end{array}\right)
$$

We write the matrix $\gamma_{k}=\left\{\gamma_{i j}^{k}\right\}_{i, j=0,1, \cdots, k}$ as,

$$
\gamma_{k}=\left(\begin{array}{cc}
\gamma_{k-1} & \overline{\gamma_{k 2}} \\
\overline{\gamma_{2 k}} & \gamma_{k k}
\end{array}\right)=\left(\begin{array}{cc}
\gamma_{00}^{k} & \widetilde{\gamma_{k 1}} \\
\widetilde{\gamma_{2 k}} & \gamma_{2 k 2}
\end{array}\right)
$$

where $\quad \overline{\gamma_{k 2}}=\left[\gamma_{0 k}, \gamma_{1 k}, \cdots, \gamma_{k-1, k}\right]^{t}, \overline{\gamma_{2 k}}=\left[\gamma_{k 0}, \gamma_{k 1}, \cdots, \gamma_{k, k-1}\right] \quad$ and $\quad \widetilde{\gamma_{k 1}}=\left[\gamma_{01}, \gamma_{02}, \cdots, \gamma_{0 k}\right], \widetilde{\gamma_{2 k}}=$ $\left[\gamma_{10}, \gamma_{20}, \cdots, \gamma_{k 0}\right]^{t}, \gamma_{2 k 2}=\left\{\gamma_{i j}^{k}\right\}_{i, j=1, \cdots, k}$.

Therefore, $T_{k-1} \overline{\gamma_{k 2}}+A_{k} \gamma_{k k}=0$ and $A_{k}^{*} \overline{\gamma_{k 2}}+\gamma_{k k}=1$. This leads to $\overline{\gamma_{k 2}}=-T_{k-1}^{-1} A_{k} \gamma_{k k}$. Hence

$$
\gamma_{k k}^{-1}=1-A_{k}^{*} T_{k-1}^{-1} A_{k}
$$

From the previous proposition we obtain the result.

Using the other representation of $T_{k}$ and $\gamma_{k}$ and the previous proposition we stablish the formula for $\gamma_{00}^{k}$.

\section{The Levinson Recurrences in an infinite dimensional setting}

In this section we extend the Levinson recurrences to the infinite dimensional setting and we derive an interesting formula for these recurrences using the generators of the defect spaces of an isometry. In this way we get a set of parameters whose norm can be interpreted as the angle between the subspaces. Similar results have been obtained in the finite dimensional setting (cf. [24] and [25]).

Proposition 4

Set $\angle_{\mathcal{M}_{p-1}}^{V_{p} \mathcal{N}_{p-1}}$ the angle between the subspaces $V_{p} \mathcal{N}_{p-1}$ and $\mathcal{M}_{p-1},\left\{\alpha_{k}\right\}_{k=1}^{\infty}$ a complete orthonormal system of the separable Hilbert space $\mathcal{G}$ and $\Lambda_{p}: \mathcal{G} \rightarrow \mathcal{G}$ the map defined by

$$
\Lambda_{p} x=\sum_{k=1}^{\infty} \sum_{l=1}^{\infty}\left\langle x, \alpha_{l}\right\rangle_{\mathcal{G}}\left\langle\zeta n_{p-1}^{l}, m_{p-1}^{k}\right\rangle_{p} \alpha_{k} .
$$

Then, this map verifies the following properties

(a) $\Lambda_{p} x=M_{p-1}^{-1} P_{\mathcal{M}_{p-1}}^{\mathcal{E}_{p-1}} V_{p} P_{\mathcal{N}_{p-1}}^{\mathcal{E}_{p-1}}\left(e_{p-1} \gamma_{p-1, p-1}^{1 / 2} x\right)$ for all $x \in \mathcal{G}$;

(b) $\left\langle\Lambda_{p} \alpha_{j}, \alpha_{i}\right\rangle_{\mathcal{G}}=\left\langle\zeta n_{p-1}^{j}, m_{p-1}^{i}\right\rangle_{p}$;

(c) $\left\|\Lambda_{p}\right\|=\cos \left(\measuredangle_{\mathcal{M}_{p-1}}^{V_{p} \mathcal{N}_{p-1}}\right)$;

Proof: In order to prove the statement (a), we use lemma 2 and part (b) of the proposition 1 to obtain,

$$
P_{\mathcal{N}_{p}}^{\mathcal{E}_{p}}\left(e_{p} \alpha_{j}\right)=\sum_{k=1}^{\infty}\left\langle e_{p} \alpha_{j}, n_{p}^{k}\right\rangle_{p} n_{p}^{k}=N_{p} \gamma_{p p}^{-1 / 2} \alpha_{j}
$$


and thus,

$$
\begin{aligned}
P_{\mathcal{M}_{p-1}}^{\mathcal{E}_{p-1}} V_{p} P_{\mathcal{N}_{p-1}}^{\mathcal{E}_{p-1}}\left(e_{p-1} \alpha_{j}\right) & =P_{\mathcal{M}_{p-1}}^{\mathcal{E}_{p-1}} V_{p} N_{p-1} \gamma_{p-1, p-1}^{-1 / 2} \alpha_{j} \\
& =M_{p-1} \sum_{k=1}^{\infty} \sum_{l=1}^{\infty}\left\langle\gamma_{p-1, p-1}^{-1 / 2} \alpha_{j}, \alpha_{l}\right\rangle_{\mathcal{G}}\left\langle V_{p} n_{p-1}^{l}, m_{p-1}^{k}\right\rangle_{p} \alpha_{k}
\end{aligned}
$$

From (8), we get

$$
P_{\mathcal{M}_{p-1}}^{\mathcal{E}_{p-1}} V_{p} P_{\mathcal{N}_{p-1}}^{\mathcal{E}_{p-1}}\left(e_{p-1} \alpha_{j}\right)=M_{p-1} \Lambda_{p} \gamma_{p-1, p-1}^{-1 / 2} \alpha_{j}
$$

which proves the result.

The part (b) holds under the hypothesis, $\left\{\alpha_{k}\right\}_{k=1}^{\infty}$ is a complete orthonormal system of $\mathcal{G}$,

$$
\left\langle\Lambda_{p} \alpha_{j}, \alpha_{i}\right\rangle_{\mathcal{G}}=\left\langle\sum_{k=1}^{\infty} \sum_{l=1}^{\infty}\left\langle\alpha_{j}, \alpha_{l}\right\rangle_{\mathcal{G}}\left\langle\zeta n_{p-1}^{l}, m_{p-1}^{k}\right\rangle_{p} \alpha_{k}, \alpha_{i}\right\rangle_{\mathcal{G}}=\left\langle\zeta n_{p-1}^{j}, m_{p-1}^{i}\right\rangle_{p}
$$

To prove the statement (c), let $x \in \mathcal{N}_{p-1}, y \in \mathcal{M}_{p-1}$ with $\|x\|_{p-1}=1$ and $\|y\|_{p-1}=1$. Thus, $x=\sum_{i=1}^{\infty} x_{i} n_{p-1}^{i}$, $y=\sum_{j=1}^{\infty} y_{j} m_{p-1}^{j}$, where $\sum_{i=1}^{\infty}\left|x_{i}\right|^{2}=1$ and $\sum_{j=1}^{\infty}\left|y_{i}\right|^{2}=1$. From the definition of the angle between two subspaces and the previous results, we have,

$$
\begin{aligned}
\cos \left(\angle_{\mathcal{M}_{p-1}}^{V_{p} \mathcal{N}_{p-1}}\right) & =\sup \left\{\left|\langle\zeta x, y\rangle_{p}\right|:\|x\|_{p-1}=1,\|y\|_{p-1}=1, x \in \mathcal{N}_{p-1}, y \in \mathcal{M}_{p-1}\right\} \\
& =\sup \left\{\left|\sum_{i=1}^{\infty} \sum_{j=1}^{\infty} x_{i} \overline{y_{j}}\left\langle\zeta n_{p-1}^{i}, m_{p-1}^{j}\right\rangle_{p}\right|: \sum_{i=1}^{\infty}\left|x_{i}\right|^{2}=1, \sum_{j=1}^{\infty}\left|y_{j}\right|^{2}=1\right\} \\
& =\sup \left\{\left|\sum_{i=1}^{\infty} \sum_{j=1}^{\infty} x_{i} \overline{y_{j}}\left\langle\Lambda_{p} \alpha_{i}, \alpha_{j}\right\rangle_{\mathcal{G}}\right|: \sum_{i=1}^{\infty}\left|x_{i}\right|^{2}=1, \sum_{j=1}^{\infty}\left|y_{j}\right|^{2}=1\right\} \\
& =\sup \left\{\left|\left\langle\Lambda_{p} u, v\right\rangle_{\mathcal{G}}\right|:\|u\|_{\mathcal{G}}^{2}=1,\|v\|_{\mathcal{G}}^{2}=1\right\} \\
& =\left\|\Lambda_{p}\right\| .
\end{aligned}
$$

Corollary 1

Let $\Lambda_{p}$ the operator defined by (8). Then,

$$
\Lambda_{p}=\sum_{m=1}^{p} \sum_{n=0}^{p-1} M_{p-1, n}^{*} R_{m-n} N_{p-1, m-1} .
$$

Moreover,

$$
\begin{aligned}
\Lambda_{p}=\Lambda_{p-1}+M_{p-1,0} R_{p} N_{p-1, p-1}+M_{p-1, p-1}^{*} & \left(\sum_{m=1}^{p-1} R_{m-p+1} N_{p-1, m-1}\right) \\
& +\left(\sum_{n=1}^{p-1} M_{p-1, n}^{*} R_{p-n}\right) N_{p-1, p-1}
\end{aligned}
$$

Proof: To stablish (10) we note that,

$$
\left\langle e_{1} n_{p-1}^{l}, m_{p-1}^{k}\right\rangle_{p}=\left\langle e_{1} N_{p-1} \alpha_{l}, M_{p-1} \alpha_{k}\right\rangle_{p}=\sum_{m=1}^{p} \sum_{n=0}^{p-1}\left\langle M_{p-1, n}^{*} R_{m-n} N_{p-1, m-1} \alpha_{l}, \alpha_{k}\right\rangle_{\mathcal{G}} .
$$


Therefore,

$$
\begin{aligned}
\Lambda_{p} x & =\sum_{k=1}^{\infty} \sum_{l=1}^{\infty}\left\langle x, \alpha_{l}\right\rangle_{\mathcal{G}}\left\langle\zeta n_{p-1}^{l}, m_{p-1}^{k}\right\rangle_{p} \alpha_{k} \\
& =\sum_{k=1}^{\infty} \sum_{l=1}^{\infty}\left\langle x, \alpha_{l}\right\rangle_{\mathcal{G}} \sum_{m=1}^{p} \sum_{n=0}^{p-1}\left\langle M_{p-1, n}^{*} R_{m-n} N_{p-1, m-1} \alpha_{l}, \alpha_{k}\right\rangle_{\mathcal{G}} \alpha_{k} \\
& =\sum_{m=1}^{p} \sum_{n=0}^{p-1} M_{p-1, n}^{*} R_{m-n} N_{p-1, m-1} x .
\end{aligned}
$$

The recursion for $\Lambda_{p}$ is a direct consequence of (10).

Now, we need to define the following operators to simplify the notation in the main result of this work,

$$
W_{p}=\gamma_{p-1, p-1}^{-1 / 2} \gamma_{p p}^{1 / 2}, \quad S_{p}=\left(\gamma_{00}^{p-1}\right)^{-1 / 2}\left(\gamma_{00}^{p}\right)^{1 / 2}
$$

From the proposition 3 these operators can be computed from $M_{p-1}, N_{p-1}$ and the sequence $R_{1}, \cdots, R_{p}$.

Theorem 1

(Levinson algorithm) Let $M_{p}$ y $N_{p}$ the operators trigonometric polynomials defined by (6) and $\Lambda_{p}$ as in (8). Then, for every $p \in \mathbb{N}$ the following recurrences are true,

$$
\begin{gathered}
N_{p}=\left(\zeta N_{p-1}-M_{p-1} \Lambda_{p}\right) W_{p}, \quad N_{0}=1 \\
M_{p}=\left(M_{p-1}-\zeta N_{p-1} \Lambda_{p}^{*}\right) S_{p}, \quad M_{0}=1 .
\end{gathered}
$$

Proof: First, let $\left\{\alpha_{k}\right\}_{k=1}^{\infty}$ a complete orthonormal system of the separable Hilbert space $\mathcal{G}$ and note that $e_{p} \alpha_{j}=V_{p}\left(e_{p-1} \alpha_{j}\right)$ and $e_{p-1} \alpha_{j}=P_{\mathcal{D}_{p}}^{\mathcal{E}_{p-1}}\left(e_{p-1} \alpha_{j}\right)$. From lemma $1, \mathcal{E}_{p-1}=\mathcal{D}_{p}=\mathcal{D}_{p-1} \oplus \mathcal{N}_{p-1}$. Hence,

$$
P_{\mathcal{D}_{p}}^{\mathcal{E}_{p}}\left(e_{p} \alpha_{j}\right)=V_{p} P_{\mathcal{D}_{p-1}}^{\mathcal{E}_{p-1}}\left(e_{p-1} \alpha_{j}\right)+P_{\mathcal{D}_{p}}^{\mathcal{E}_{p}} V_{p} P_{\mathcal{N}_{p-1}}^{\mathcal{E}_{p-1}}\left(e_{p-1} \alpha_{j}\right)
$$

Second, from of the orthogonal decompositions $\mathcal{D}_{p}=\mathcal{R}_{p-1} \oplus \mathcal{M}_{p-1}$ and $\mathcal{R}_{p}=\mathcal{R}_{p-1} \oplus V_{p} \mathcal{N}_{p-1}$, we have,

$$
\begin{aligned}
P_{\mathcal{D}_{p}}^{\mathcal{E}_{p}} V_{p} P_{\mathcal{N}_{p-1}}^{\mathcal{E}_{p-1}}\left(e_{p-1} \alpha_{j}\right. & =P_{\mathcal{R}_{p-1} \oplus \mathcal{M}_{p-1}}^{\mathcal{E}_{p}} V_{p} P_{\mathcal{N}_{p-1}}^{\mathcal{E}_{p-1}}\left(e_{p-1} \alpha_{j}\right) \\
& =P_{\mathcal{M}_{p-1}}^{\mathcal{E}_{p-1}} V_{p} P_{\mathcal{N}_{p-1}}^{\mathcal{E}_{p-1}}\left(e_{p-1} \alpha_{j}\right) .
\end{aligned}
$$

Substituting (13) into (12), we obtain,

$$
P_{\mathcal{D}_{p}}^{\mathcal{E}_{p}}\left(e_{p} \alpha_{j}\right)=V_{p} P_{\mathcal{D}_{p-1}}^{\mathcal{E}_{p-1}}\left(e_{p-1} \alpha_{j}\right)+P_{\mathcal{M}_{p-1}}^{\mathcal{E}_{p-1}} V_{p} P_{\mathcal{N}_{p-1}}^{\mathcal{E}_{p-1}}\left(e_{p-1} \alpha_{j}\right)
$$

Again, using the orthogonal decomposition, $\mathcal{D}_{p+1}=\mathcal{D}_{p} \oplus \mathcal{N}_{p}$ we get $P_{\mathcal{N}_{p}}^{\mathcal{E}_{p}}\left(e_{p} \alpha_{j}\right)=e_{p} \alpha_{j}-P_{\mathcal{D}_{p}}^{\mathcal{E}_{p}}\left(e_{p} \alpha_{j}\right)$. This operator is called the forward innovation operator. Now, we can rewrite (14) as,

$$
P_{\mathcal{N}_{p}}^{\mathcal{E}_{p}}\left(e_{p} \alpha_{j}\right)=V_{p} P_{\mathcal{N}_{p-1}}^{\mathcal{E}_{p-1}}\left(e_{p} \alpha_{j}\right)-P_{\mathcal{M}_{p-1}}^{\mathcal{E}_{p-1}} V_{p} P_{\mathcal{N}_{p-1}}^{\mathcal{E}_{p-1}}\left(e_{p-1} \alpha_{j}\right)
$$

Finally, from proposition 4 we have the first recurrence of (11),

$$
N_{p} \gamma_{p p}^{-1 / 2} \alpha_{j}=\zeta N_{p-1} \gamma_{p-1, p-1}^{-1 / 2} \alpha_{j}-M_{p-1} \Lambda_{p} \gamma_{p-1, p-1}^{-1 / 2} \alpha_{j} .
$$

For the other recursion we need the orthogonal decomposition $\mathcal{R}_{p}=\mathcal{R}_{p-1} \oplus V_{p} \mathcal{N}_{p-1}$. Thus,

$$
P_{\mathcal{R}_{p}}^{\mathcal{E}_{p}}\left(e_{0} \alpha_{j}\right)=P_{\mathcal{R}_{p-1}}^{\mathcal{E}_{p}}\left(e_{0} \alpha_{j}\right)+P_{V_{p} \mathcal{N}_{p-1}}^{\mathcal{E}_{p}}\left(e_{0} \alpha_{j}\right)
$$


Now, from the orthogonal decomposition $\mathcal{E}_{p}=\mathcal{R}_{p} \oplus \mathcal{M}_{p}$ we have, $P_{\mathcal{M}_{p}}^{\mathcal{E}_{p}}\left(e_{0} \alpha_{j}\right)=e_{0} \alpha_{j}-P_{\mathcal{R}_{p}}^{\mathcal{E}_{p}}\left(e_{0} \alpha_{j}\right)$. This operator is called the backward innovation operator. Thus, we can rewrite (15) as

$$
P_{\mathcal{M}_{p}}^{\mathcal{E}_{p}}\left(e_{0} \alpha_{j}\right)=P_{\mathcal{M}_{p-1}}^{\mathcal{E}_{p}}\left(e_{0} \alpha_{j}\right)-P_{V_{p} \mathcal{N}_{p-1}}^{\mathcal{E}_{p}}\left(e_{0} \alpha_{j}\right)
$$

From proposition 1 and lemma 2, we have $P_{\mathcal{M}_{p}}^{\mathcal{E}_{p}}\left(e_{0} \alpha_{j}\right)=M_{p}\left(\gamma_{00}^{p}\right)^{-1 / 2} \alpha_{j}$. Therefore,

$$
M_{p}\left(\gamma_{00}^{p}\right)^{-1 / 2} \alpha_{j}=M_{p-1}\left(\gamma_{00}^{p-1}\right)^{-1 / 2} \alpha_{j}-P_{V_{p} \mathcal{N}_{p-1}}^{\mathcal{E}_{p}}\left(e_{0} \alpha_{j}\right)
$$

It remains to obtain $P_{V_{p} \mathcal{N}_{p-1}}^{\mathcal{E}_{p}}\left(e_{0} \alpha_{j}\right)$. Note that from proposition 1, lemmas 1 and 2 and (8),

$$
\begin{aligned}
P_{V_{p} \mathcal{N}_{p-1}}^{\mathcal{E}_{p}}\left(e_{0} \alpha_{j}\right) & =P_{V_{p} \mathcal{N}_{p-1}}^{\mathcal{E}_{p}}\left(P_{\mathcal{R}_{p-1}}^{\mathcal{E}_{p}}\left(e_{0} \alpha_{j}\right)+P_{\mathcal{M}_{p-1}}^{\mathcal{E}_{p}}\left(e_{0} \alpha_{j}\right)\right) \\
& =P_{V_{p} \mathcal{N}_{p-1}}^{\mathcal{E}_{p}} P_{\mathcal{M}_{p-1}}^{\mathcal{E}_{p}}\left(e_{0} \alpha_{j}\right)=V_{p} N_{p-1} \Lambda_{p}^{*}\left(\gamma_{00}^{p-1}\right)^{-1 / 2} .
\end{aligned}
$$

Substituting this expression into (16), we conclude that

$$
M_{p}\left(\gamma_{00}^{p}\right)^{-1 / 2}=M_{p-1}\left(\gamma_{00}^{p-1}\right)^{-1 / 2}-\zeta N_{p-1} \Lambda_{p}^{*}\left(\gamma_{00}^{p-1}\right)^{-1 / 2}
$$

Which proves the main result of this work.

\section{Conclusions and Discussions}

In this work we have obtained a new version of the Levinson algorithm in infinite dimension setting using a geometrical approach. This result, together with the lower and upper triangular factorization of the inverse for block Toeplitz matrix $T_{p}$ allows us to solve efficiently system of equations $T_{p} X_{p}=Y_{p}$ where $X_{p}, Y_{p} \in \mathcal{G}^{p+1}$ , recursively computing the solution of the system of increasing size $T_{k} X_{k}=Y_{k}$. Additionally, the parameters obtained can be interpreted in terms of angles between subspaces. This generalizes the result obtained in the finite dimensional case where this parameters can be identified with the partial autocorrelation coefficients. Indeed, if we assume that $1=R_{0}, \ldots, R_{p}$ are the scalar partial autocorrelation coefficients of a second order stationary stochastic process $X=\left\{X_{k}\right\}_{k \in \mathbb{Z}}$ this sequence is strictly positive definite. From [25] we know that the map $X_{-j} \rightarrow e_{j}, j \in \mathbb{Z}$ establishes a unitary automorphism between $H_{X}=\overline{\operatorname{Span}\left\{X_{j}: j \in \mathbb{Z}\right\}}$ (the closure of the space generated by the process) and $L^{2}(f d t)$ where $f$ is the spectral density of the process. Now, let $l, k \in \mathbb{N}, k>l$ and $H_{l, k}:=\operatorname{Span}\left\{X_{-n}\right\}_{n=l}^{k}$ subspaces of $H^{X}$. We define the innovations by

$$
\epsilon_{p}=X_{-p}-P_{H_{1, p-1}} X_{-p}, \quad \epsilon_{p}^{*}=X_{0}-P_{H_{1, p-1}} X_{0}
$$

and they verify

$$
\frac{\left\langle\epsilon_{p}, \epsilon_{p}^{*}\right\rangle_{H^{X}}}{\left\langle\epsilon_{p}, \epsilon_{p}\right\rangle_{H^{X}}^{1 / 2}\left\langle\epsilon_{p}^{*}, \epsilon_{p}^{*}\right\rangle_{H^{X}}^{1 / 2}}=\left\langle V_{p} n_{p-1}, m_{p-1}\right\rangle_{p}=\cos \left(\left\langle_{\mathcal{M}_{p-1}}^{V_{p} \mathcal{N}_{p-1}}\right)=\left\|\Lambda_{p}\right\| .\right.
$$

These parameters are called partial de autocorrelation coefficients and are obtained from the Levinson algorithm. When the dimension is $q$ we have a similar result. More details can be found in [24].

In this paper we show that the parameters $\Lambda_{p}$ have the same interpretation: $\left\|\Lambda_{p}\right\|=\cos \left(\angle_{\mathcal{M}_{p-1}}^{V_{p} \mathcal{N}_{p-1}}\right)$.

We have obtained from Corollary 1 that these parameters can be computed recursively and there exists a one to one correspondence between these parameter and the sequence of operators $\left\{R_{k}\right\}_{k=1}^{p}$.

Finally, the geometrical technique obtained in this work could be useful to solve extension problems in Statistics and for prediction problems in an infinite dimensional setting. 


\section{REFERENCES}

1. R. Arocena, Extensiones unitarias de isometrías, entropía máxima y parámetros de Schur de un proceso estacionario, Spanish, Actas III Congreso Latinoamericano: Prob. y Est. Mat., 1-8, 1990.

2. M. Bakonyi and T. Constantinescu, Schur's algorithm and several applications, Pitman Research Notes in Mathematics Series, 261, 1992.

3. D. Bosq, Linear Processes in Function Spaces, Lecture Notes in Statistics, Springer, 2000.

4. D. Bosq, Computing the best linear predictor in a Hilbert space. Applications to general ARMAH processes, Journal of Multivariate Analysis, 124, 436-450, 2014.

5. P. Brockwel and R. Davis, Time Series: Theory and Methods. Second Edition, Springer Series in Statistics, New York, 2006.

6. J. Burg, Maximum Entropy Spectral Analysis, Thesis for the degree of Doctor of Philosophy, Department of Geophysics, Stanford University, California, 1975.

7. G. Castro, Coeficientes de réflexion géneralisés. Extension de covariances multidimensionalles et autres applications, Thesis for the degree of Doctor of Philosophy, Université d'Orsay, 1996.

8. J. Claerbout, Fundamentals of geophysical data processing, McGraw-Hill, 1976.

9. B. Choi, Multivariable maximum entropy spectrum, Journal of Multivariate Analysis, 46, 56-60, 1993.

10. S. Degerine, Canonical partial autocorrelation function of a multivariate time series, Annals of Statistics, 18, No. 2, 961-971, 1990.

11. P. Delsarte, Y. Genin and Y. Kamp, Orthogonal polynomial matrices on the unit circle, IEEE Trans. Circuits Syst., CAS-25, 149-160, 1978.

12. P. Delsarte, Y. Genin y Y. Kamp, Schur parametrization of positive definite block-Toeplitz system, Siam J. Appl. Math, 36, 34-46, 1979.

13. D. Dudgeon and R. Mersereau, Multidimensional Digital Signal Processing, Prentice Hall, Englewood Cliffs, New Jersey, 1984.

14. M. Dugast, G. Bouleux and E. Marcon, Representation and characterization of nonstationary processes by dilation operator and induced shape space manifolds, Entropy, 20, No. 9, 2018.

15. H. Dym, Hermitian block Toeplitz matrices, orthogonal polynomials, reproducing kernel, Pontryagin spaces, interpolation and extension, Operator Theory: Advances and Applications, 34, 1988.

16. C. Foias and A. Frazho, The commutant lifting approach to interpolation problems, Operator Theory: Advances and Applications, 44, 1990.

17. Ya. Geronimus, Orthogonal Polynomials, New York Consultants Bureau, 1961.

18. T. Kailath, A theorem of I. Schur and its impact on modern signal processing, Operator Theory: Advances and Applications, 18, 9-29, 1986.

19. H. Landau, Maximum entropy and the moment problem, Bull. Am. Math. Soc. 16, No. 1, 47-77, 1987.

20. N. Levinson, The Wiener RMS (root-mean-square) error criterion in filter design and prediction, J. Math. Phys., 25, 261-278, 1947.

21. M. Luz and M. Moklyachuck, Filtering problem for functionals of stationary sequences, Statistics, Optimization and Information Computing, 4, No. 1, 68-83, 2016.

22. P. Masani, Recent trends in multivariate prediction theory, Multivariate Anal., Academic Press, New York, 351-382, 1966.

23. J. Marcano, S. Infante and L. Sánchez, On a generalization of maximum entropy of Burg, Revista de Matemáticas: Teoría y Aplicaciones, 24, No, 1, 97-113, 2017.

24. J. Marcano and M. Morán, The Arov-Grossman model and Burg multivariate entropy, J. Fourier Anal. and Applications, Vol. 9, No. 6, 623-647, 2003.

25. J. Marcano and M. Morán, The Arov-Grossman model and Burg's entropy, in Recent Advances in Applied Probability, Ed Baeza-Yates R., Glaz J.,Gzyl H., Husler J. and Palacios J.L., 329-348, 2005.

26. S. Marple, Digital Spectral Analysis with applications, Prentice Hall, Englewood Cliffs, New Jersey, 1987.

27. M. Moklyachuck, Minimax-robust estimation problem for stationary stochastic sequences, Statistics, Optimization and Information Computing, 3, No. 4, 348-419, 2015.

28. M. Morf, A. Viera and T. Kailath, Covariance characterization by partial autocorrelation matrices, Annals of Statistics, 6, No. 3, 643-648, 1978

29. M. Morf, A. Vieira, D. Lee and T. Kailath, Recursive multichannel maximum entropy method, IEEE Transaction on Geoscience Electronics, 16(2), 85-94, 1978.

30. B. Sz-Nagy and C. Foias, Harmonic Analysis of Operators on Hilbert Spaces, North-Holland, Amsterdan, 1970.

31. I. Schur, On power series which are bounded in the interior of the unit circle I, English translation in I. Schur Method in operator theory and signal processing, Op. theory: Adv. and Appl., Ed. I. Gohberg, 18, 31-59, 1986.

32. I. Schur, On power series which are bounded in the interior of the unit circle II, English translation in I. Schur Method in operator theory and signal processing, Op. theory: Adv. and Appl., Ed. I. Gohberg, 18, 61-88, 1986.

33. P. Whittle, On the fitting of multivariate autoregressions, and the approximate canonical factorization of a spectral density matrix, Biometrika, 50, 129-134, 1963.

34. R. Wigging and E. Robinson, Recursive solution to the multichannel filtering problem, J. Geophys. Res., 70, 1885-1891, 1965.

35. H. Woerdeman, Matrix and operator extensions, Thesis for the degree of Doctor of Philosophy, Amsterdam, 1989. 